\title{
LC-UV and LC-MS methods for the determination of domoic acid
}

\author{
Philipp Hess, Steven Morris, Lesley A. Stobo, Nigel A. Brown, \\ John D.G. McEvoy, Glenn Kennedy, Paul B. Young, Deirdre Slattery, Evin \\ McGovern, Terry McMahon, Susan Gallacher
}

Under European legislation, domoic acid (DA), the main constituent of amnesic shellfish poisoning, is monitored to protect the shellfish consumer. To ensure comparability amongst analytical data, it was deemed necessary to undertake performance assessments of the methods conducted by monitoring laboratories of the United Kingdom and Ireland.

In phase I of a two-phase inter-comparison, three laboratories used high-performance liquid chromatography and ultraviolet detection (HPLC-UV). Concentration data for a DA standard solution, a crude extract of whole scallops and a scallop-homogenate fell within internationally accepted limits, demonstrating good agreement for these matrices. Between-laboratory analyses of a scallop gonad showed a higher variation $(>16 \%)$.

In phase II, a second gonad homogenate containing DA one order of magnitude higher in concentration gave results acceptable to internationally set criteria.

The efficiency of the strong anion-exchange cartridges used in sample-extract clean-up should be monitored as part of a laboratory quality control system.

From a recovery study, it is suggested that recovery correction should also be applied.

There was no difference in the quantitation of DA in standard solutions or shellfish using either LC-UV or LC with mass spectrometric (MS) detection, and between-laboratory MS data for a gonad homogenate were also equivalent.

Variations of the published method practised by the monitoring laboratories were found not to compromise results, thus demonstrating an acceptable degree of ruggedness, as well as comparability between the participants.

Keywords: Domoic acid LC-MS LC-UV Ruggedness Scallops Standard solution

\section{Introduction}

Philipp Hess, Evin McGovern, Terry McMahon

Marine Institute, Galway Technical Park, Parkmore West, Galway, Ireland

\section{Steven Morris}

Centre for Environment, Fisheries and Aquaculture Science, Remembrance Avenue

Burnham-on-Crouch, Essex CMO 8HA, UK

Lesley A. Stobo, Nigel A. Brown, Susan Gallacher

Fisheries Research Services, Marine Laboratory Aberdeen, Victoria Road, Aberdeen AB11 9DB, UK

John D.G. McEvoy, Glenn Kennedy, Paul B. Young, Deirdre Slattery

Department of Agriculture and Rural Development Northern Ireland, Veterinary Sciences Division

Stoney Road, Stormont, Belfast BT4 3SD, UK
Amnesic shellfish poisoning (ASP) toxins are produced by microalgae (e.g., Pseudonitzschia spp.) and can accumulate in shellfish. Consumption of toxic shellfish may cause a number of effects in humans, including vomiting, diarrhea and permanent short-term memory loss [1].

European Union (EU) legislation [2] was amended in 1997 [3], and adopted in the United Kingdom (UK) in 1998, to include domoic acid (DA), the main constituent of ASP toxins, in the suite of biotoxins to be determined in the regulatory monitoring of shellfish. The amendment requires shellfish to contain less than $20 \mathrm{mg} / \mathrm{kg}$ of DA as analyzed by high-performance liquid chromatography (HPLC) with ultraviolet (UV) detection; however, no specific procedure has been officially validated or recommended by the EU. 
The International Scientific Community has proposed two methods $[4,5]$. The suitability of these methods is being investigated by the European Committee on Normalization (CEN) and by a EU working group. Irish and UK laboratories involved in the regulatory monitoring of shellfish poisons have since adopted the procedure of Quilliam et al. [4]. A performance assessment was deemed necessary to ensure comparability amongst the monitoring laboratories involved in the routine analyses of ASP, so inter-laboratory comparisons were undertaken involving four laboratories whereby concentration data was reviewed on the basis of applied methodologies.

For the purposes of this inter-comparison study reported here, three biotoxin-monitoring laboratories (LAB-1, LAB-2 and LAB-3) were included along with LAB-4, which participated to develop LC with mass spectrometric (LC-MS) method of analysis. The study consisted of two phases. Thus, this article describes results of an inter-laboratory comparison study, as performed by the four laboratories, of the HPLC-UV and LC-MS methods used for the detection and quantification of DA in standard solutions and in real shellfish samples naturally incurred with DA.

\section{Inter-laboratory performance studies}

External assessments of the quality of the results generated by individual laboratories and in the form of interlaboratory comparisons satisfy the requirement to demonstrate comparability of analytical data. By centrally distributing samples, assessments of performance in the inter-laboratory studies were made possible, and the participation in such comparisons proved necessary in method development and refinement, as well as validation.

\subsection{Sample management}

LAB-1 prepared and distributed all of the standard solutions, scallop-tissue homogenates, and crude extracts used in both phases of this study. A certified reference material [CRM, MUS1-B; National Research Council (NRC) Canada)] containing DA was also examined in phase II. To assess the stability of the samples over the study periods, the DA contents in aliquots of standard solutions, extracts and homogenates were also determined by LAB-1 prior to sample dispatch, and following receipt of the results from each of the participants.

\subsection{Statistical analysis and criteria}

A comparison of concentration data sets generated by HPLC-UV and LC-MS were evaluated statistically to examine if they were significantly different from each other. This was carried out using a $t$-test or a one-way ANOVA on ranks according to Kruskal-Wallis. Statistical analysis of the data was also conducted in accordance with "Quality Assurance of Information for Marine Environmental Monitoring" (QUASIMEME) [6,7], an international proficiency-testing scheme. The scheme assesses the proficiency of participating laboratories by comparing their results with assigned target values. For each analysis, a "maximum allowable error" (MAE) [8], is defined as the sum of proportional and constant errors. Data are then assessed as "satisfactory", "questionable" or "unsatisfactory" according to the degree to which they deviate from the assigned or target value. For instance, at a MAE value of $12.5 \%$, data within $25 \%$ of the assigned value would be assessed as "satisfactory". Target values were established as either the nominal values for standard solutions or the mean of the participant's results for DA content in extracts and tissues.

The statistical model used in this study was based on the QUASIMEME model using proportional errors and did not include any allowance for constant errors. The proportional errors in QUASIMEME inter-comparison exercises are generally between $6 \%$ and $12.5 \%$. Since there are no agreed international parameters set for the quality of amnesic shellfish poison (ASP) analyses and, due to the potential implications to public health of DA as a food contaminant, the MAEs set in this study were stricter than those utilized by QUASIMEME. Values also varied depending on the number of sample-processing steps used by the participants during extract preparation. Table 1 details the MAE values assigned for each sample type analyzed in phases I and II of the interlaboratory exercises.

Table 1. Maximum allowable error (MAE) values for different sample matrices analyzed in the inter-comparison exercises

\begin{tabular}{|c|c|c|c|}
\hline Sample matrix & MAE (\%) & $\begin{array}{l}\text { Definition of satisfactory } \\
\text { data based on assigned value (\%) }\end{array}$ & $\begin{array}{l}\text { Number of processing steps for } \\
\text { sample preparation by participants }\end{array}$ \\
\hline Standards and crude extract & 3.13 & 6.25 & 0 \\
\hline Crude extract processed through SAX clean up & 4.7 & 9.38 & 1 \\
\hline Tissue homogenates $^{a}$ & 6.25 & 12.5 & 2 \\
\hline
\end{tabular}




\section{Phase I}

\subsection{Preparation of standard solutions, crude scallop extracts and homogenates}

Standard solutions containing DA were prepared by weight and by dilution of the certified reference standard (DACS-1C; NRC, Canada) in 10\% aqueous acetonitrile. Aliquots $(0.5 \mathrm{~mL})$ were then transferred to $2 \mathrm{~mL}$ amber ampoules and sealed under nitrogen. The nominal DA concentration was $2 \mu \mathrm{g} / \mathrm{mL}$, which the participants did not know. Ampoules were stored at $2-8^{\circ} \mathrm{C}$. Crude extracts were prepared from homogenized scallops obtained from the Scottish West Coast in 1999. These methanol/water extracts were filtered with $0.45-\mu \mathrm{m}$ syringe filters and combined, and aliquots $(0.8 \mathrm{~mL})$ were then transferred to amber ampoules, sealed under nitrogen, and stored at $2-8^{\circ} \mathrm{C}$. Both whole-flesh samples (including all of the scallop tissue) and gonads were isolated from the shells, homogenized and stored at $-20^{\circ} \mathrm{C}$. A sub-sample of gonad and whole animal was thawed thoroughly, autoclaved for $20 \mathrm{~min}\left(120^{\circ} \mathrm{C}\right)$, and then homogenized for 15 min using an Ultra Turrax. Aliquots of each homogenate were transferred to $7-\mathrm{mL}$ bijous, and stored at $-20^{\circ} \mathrm{C}$ on the same day.

The three participants (LAB-1, LAB-2 and LAB-3) manipulated and analyzed standard solutions, shellfish extracts and homogenates according to Quilliam et al. [4] with numerous variations; these are summarized in Tables 2 and 3. All participants in both phases of the study used dilutions of the certified reference standard of DA as calibration standards.

\subsection{Investigations of sample stability and homogeneity} For both phases I and II, LAB-1 analyzed all samples at the start (data represented as LAB-1-1) and at the conclusion (LAB-1-2) of each exercise. The concentration data derived from the analyses of the DA standard solutions, crude and cleaned-up scallop extracts and the whole-scallop-homogenate were within the MAE (Figs. 1 and 2).

However, and with reference to Fig. 2(b), mean concentration data for the scallop gonad fell outside the MAE value (see LAB-1-1).

In phase II, and for the analyses of the gonad sample, there was no significant difference in the DA content at the start (LAB-1-1) and end (LAB-1-2) of the trial (Figs. 4(a) and (b)). This suggests that the distributed samples were stable over the inter-comparison period, and any differences observed in the data can be interpreted as between-laboratory differences.

Homogeneity of the scallop gonads and whole-flesh samples was determined following their preparation and prior to the commencement of phase I. The DA plus its epimer, epi-DA, content of the sub-samples $(n=6)$ for each sample type was determined by HPLC-UV.

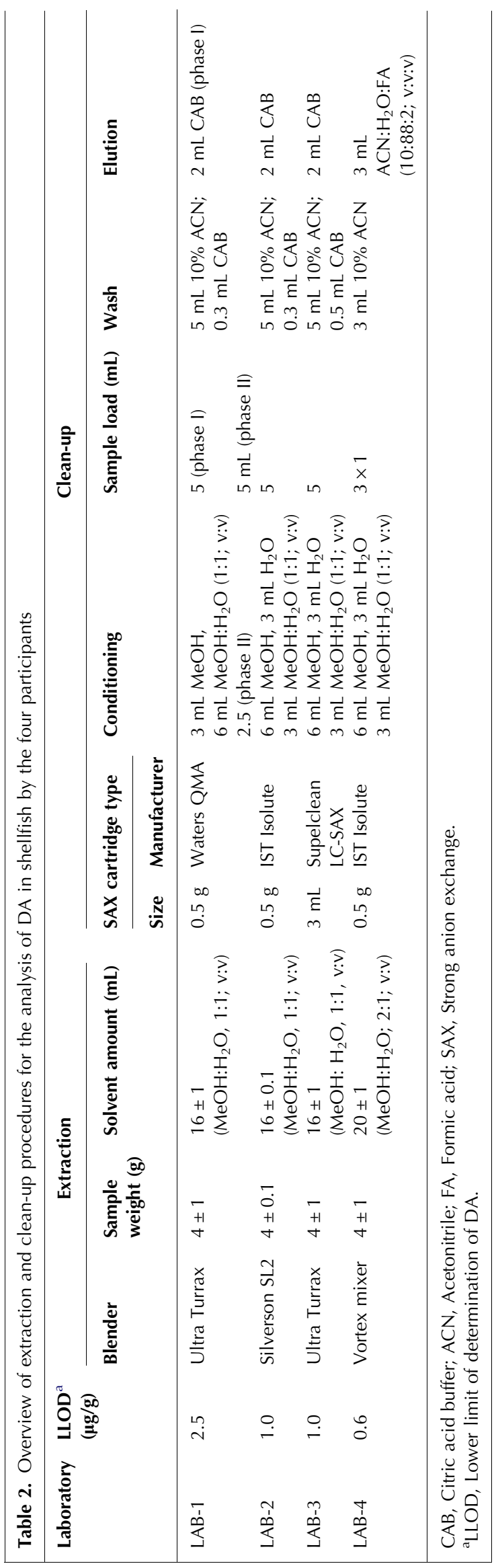


Table 3. HPLC details for the analysis of DA, as carried out by each participating laboratory

\begin{tabular}{|c|c|c|c|c|c|c|c|}
\hline \multirow{2}{*}{$\begin{array}{l}\text { Laboratory } \\
\text { (detection system }\end{array}$} & \multicolumn{2}{|c|}{ Mobile phase } & \multicolumn{2}{|c|}{ HPLC column type } & \multicolumn{2}{|c|}{ Calibration } & \multirow{2}{*}{$\begin{array}{l}\text { DA } \\
\text { \& epi-DA } \\
\text { separation }\end{array}$} \\
\hline & $\mathrm{ACN}(\%)$ & Additive $^{b}$ & Guard & Analytical & Levels $^{\mathrm{a}}$ & Range $(\mu \mathrm{g} / \mathrm{mL})$ & \\
\hline $\begin{array}{l}\text { LAB-1 phase I } \\
\text { (LC-UV) }\end{array}$ & 10 & $0.1 \%$ TFA & $\begin{array}{l}10 \times 4.6 \mathrm{~mm}, 5 \mu \mathrm{m} \\
\text { Purosphere }\end{array}$ & $\begin{array}{l}250 \times 4.6 \mathrm{~mm}, 5 \mu \mathrm{m} \\
\text { Spherisorb ODS2 }\end{array}$ & 6 & $0.25-10$ & Yes \\
\hline $\begin{array}{l}\text { LAB-1 phase II } \\
\text { (LC-UV) }\end{array}$ & 10 & $0.1 \%$ TFA & $\begin{array}{l}10 \times 4.6 \mathrm{~mm}, 5 \mu \mathrm{m} \\
\text { Vydac } 201 \mathrm{GK} 54 \mathrm{~T}\end{array}$ & $\begin{array}{l}250 \times 4.6 \mathrm{~mm}, 5 \mu \mathrm{m} \\
\text { Vydac } 201 \mathrm{TP} 54\end{array}$ & 6 & $0.25-10$ & Yes \\
\hline $\begin{array}{l}\text { LAB-1 phase II } \\
\text { (LC-MS) }\end{array}$ & 9.5 & $\begin{array}{l}2 \mathrm{mM} \mathrm{AF} \\
+50 \mathrm{mM} \mathrm{FA}\end{array}$ & $\begin{array}{l}10 \times 2 \mathrm{~mm}, 3 \mu \mathrm{m} \text { BDS } \\
\text { Hypersil C } 8\end{array}$ & $\begin{array}{l}50 \times 2 \mathrm{~mm}, 3 \mu \mathrm{m} \text { BDS } \\
\text { Hypersil C } 8\end{array}$ & 6 & $0.25-10$ & Yes \\
\hline LAB-2 (LC-UV) & 15 & $0.1 \%$ TFA & $\begin{array}{l}4 \times 4 \mathrm{~mm}, 5 \mu \mathrm{m} \\
\text { Lichrochart RP18e }\end{array}$ & $\begin{array}{l}250 \times 4.0 \mathrm{~mm}, 5 \mu \mathrm{m} \\
\text { Lichrosphere RP18e }\end{array}$ & 1 & 10 & Yes \\
\hline LAB-3 (LC-UV) & 10 & $0.1 \%$ TFA & None & $\begin{array}{l}250 \times 4.6 \mathrm{~mm}, 10 \mu \mathrm{m} \\
\text { Vydac } 201 \mathrm{TP} 104\end{array}$ & 5 & $1-50$ & Yes \\
\hline $\begin{array}{l}\text { LAB-4 phase II } \\
\text { (LC-MS) }\end{array}$ & 12.5 & $0.1 \% \mathrm{FA}$ & $\begin{array}{l}30 \times 4.6 \mathrm{~mm}, 5 \mu \mathrm{m} \\
\text { Sphereclone ODS2 }\end{array}$ & $\begin{array}{l}250 \times 4.6 \mathrm{~mm}, 5 \mu \mathrm{m} \\
\text { Sphereclone ODS } 2\end{array}$ & 7 & $0.1-9$ & Yes \\
\hline
\end{tabular}

For the gonad sample, a mean concentration and coefficient of variation (c.v.) of $3.15 \mathrm{mg} / \mathrm{kg}$ and $3.2 \%$ were found, whereas, for the whole-flesh sample, $144 \mathrm{mg} / \mathrm{kg}$ and $11.2 \%$ were determined.

Considering the levels of DA contamination associated with each sample type and their associated percentage c.v.s, it was concluded that both samples were homogeneous and fit for the purpose of this study.

\subsection{Sample extraction and analysis by HPLC-UV}

Samples were analyzed within 10 days of receipt by the participants. Using strong anion-exchange (SAX) solid phase extraction cartridges, each participant also performed a clean-up stage on the crude-scallop extract and on their prepared extracts of the whole-scallop and gonad samples. Thus, for the crude-scallop extract, the same sample was analyzed before and after SAX clean up.

With reference to Table 1, the tissue homogenates were thawed, and 4-g aliquots were weighed into centrifuge tubes. Following the addition of $16 \mathrm{~mL}$ of methanol:water (1:1 volume:volume; v:v), extraction was carried out by blending and centrifugation. An aliquot of the supernatant was transferred to a conditioned SAX cartridge and eluted with either citric or formic acid buffer following a $10 \%$ aqueous:acetonitrile $(\mathrm{v}: \mathrm{v})$ wash. The eluent was then vortex mixed and analyzed by HPLC-UV.

\subsection{Assessment of the effect of elution volume on DA recovery using the DA CRM}

To observe potential losses during the sample-extract clean-up stage, LAB-1 also assessed the elution of DA from the SAX cartridge (QMA SAX, Waters Ltd.), as proposed by Quilliam et al. [4] The elution profile was determined by collecting $0.3-\mathrm{mL}$ fractions over a total elution volume of $6 \mathrm{~mL}$. Each fraction was then analyzed for DA by HPLC-UV. This was carried out in triplicate. The DA concentrations obtained in each fraction were then plotted against the cumulative elution volume.

\section{Phase II}

All four participants were involved in phase II, and both LAB-1 and LAB-4 deployed LC-MS techniques. Phase II involved the analysis of three DA standards (5, 2 and $1 \mu \mathrm{g} / \mathrm{mL}$ ), a scallop-gonad homogenate, and a CRM (MUS1-B). The extraction methodology and HPLC conditions are summarized in Tables 2 and 3.

LAB-4 applied a different sample-extraction procedure, which involved vortex mixing (see Table 2) for $2 \mathrm{~min}$, followed by centrifugation. The volume of the extracting solvents also differed from the other laboratories in that a larger volume $(20 \mathrm{~mL})$ was used, and the ratio of methanol-to-water was 2:1 as opposed to 1:1. Although sample clean-up was the same as the other participating laboratories in terms of using a SAX sorbent, the total volume of the crude extract that was cleaned up was only $3 \mathrm{~mL}$. Elution of DA from the SAX cartridge also differed, and incorporated $88 \%$ acetonitrile with $2 \%$ formic acid as opposed to a citric acid buffer solution.

\subsection{Analysis by LC-MS}

The LC-MS used by LAB-1 was an API 150ex mass spectrometer (PE Biosystems) equipped with a TurboIonspray source and coupled to an Agilent 1100 series LC, with quaternary pump, degasser, autosampler and column thermostat compartment (held at $20^{\circ} \mathrm{C}$ ). The LC column, guard and mobile phase used are described in 

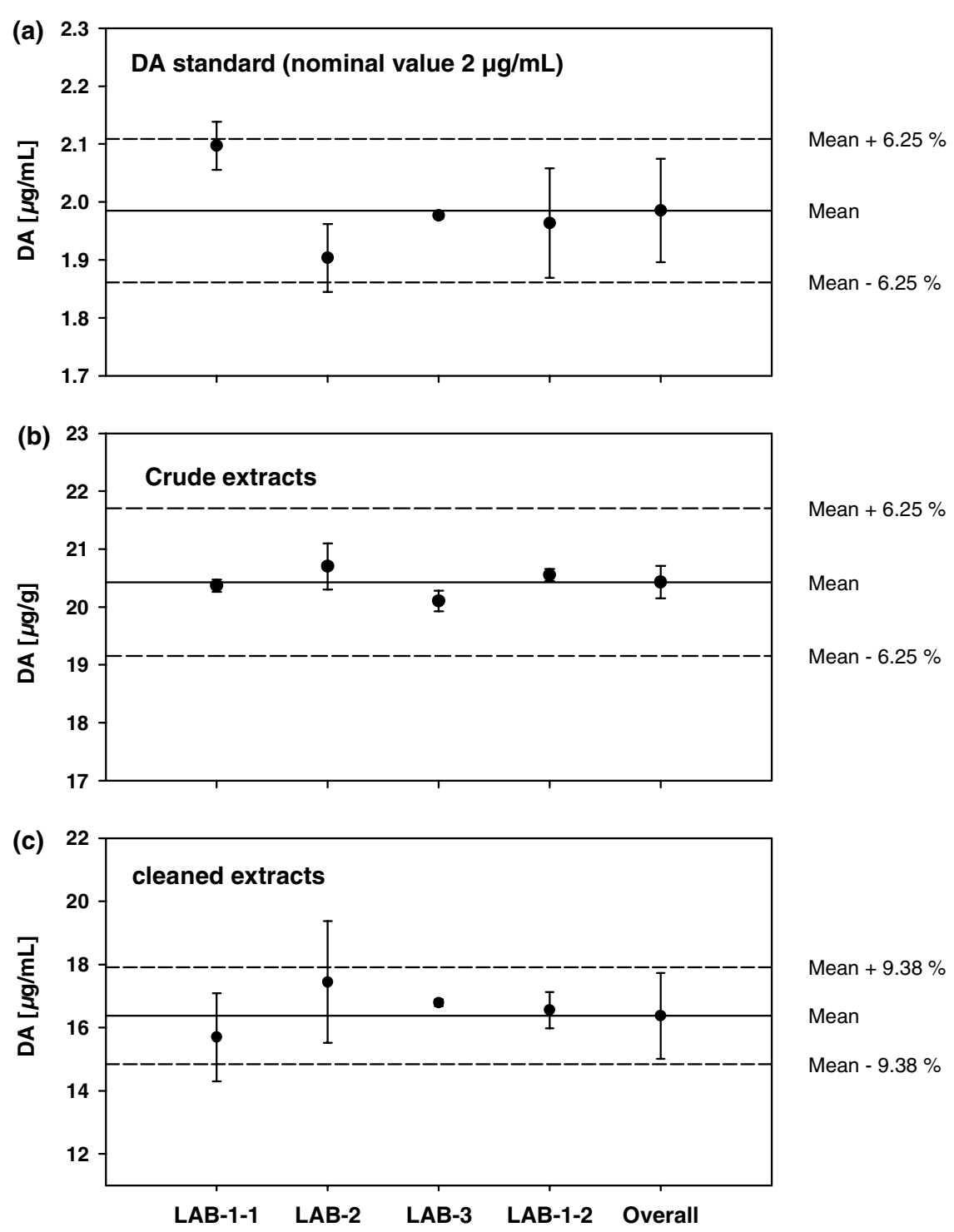

Figure 1. Phase I of the study: analysis of (a) standard DA solution, (b) crude extracts, and (c) crude extracts after SAX clean up. Error bars represent the standard deviation of the mean.

Table 2. All solvents were HPLC grade, and all reagents were of an analytical grade. Nitrogen was used as the curtain gas $(12 \mathrm{~L} / \mathrm{min})$, the spray gas $(9 \mathrm{~L} / \mathrm{min})$, and the heater gas $\left(400^{\circ} \mathrm{C} ; 7 \mathrm{~L} / \mathrm{min}\right)$. The ion-spray voltage was set at $5 \mathrm{kV}$, the orifice and ring voltage were 10 and $160 \mathrm{~V}$, respectively. Quantification was carried out using the protonated $[\mathrm{M}+\mathrm{H}]^{+}$ion of DA at $\mathrm{m} / z 312$.

For LAB-4, a single quadrupole mass spectrometer (Platform II; Micromass), interfaced with an electrospray ionization source and coupled to an HP1050 HPLC (Agilent) was used. The cone and capillary voltages were $30 \mathrm{~V}$ and $3.6 \mathrm{kV}$, and the source temperature was maintained at $160^{\circ} \mathrm{C}$.

Details of the mobile-phase composition (HPLC grade) and the LC column are given in Table 2 . External quantification was carried out, and selected ion monitoring of the $[\mathrm{M}+\mathrm{H}]^{+}$ion at $\mathrm{m} / \mathrm{z} 312.4$ was performed.

\section{Results of the inter-comparison exercises}

Concentration data and percentage c.v.s are detailed in Tables 4 and 5. Mean DA data are presented as scatter plots, and the error bars represent one standard deviation of the mean (Figs. 1-4).

\subsection{Phase I - participant performances}

The concentration data obtained by each participant fell within the MAE of $12.5 \%$ for the DA standard (Fig. 1(a)), the crude extracts of scallop (before and after SAX clean up; Figs. 1(b) and (c)), and the whole-scallop 


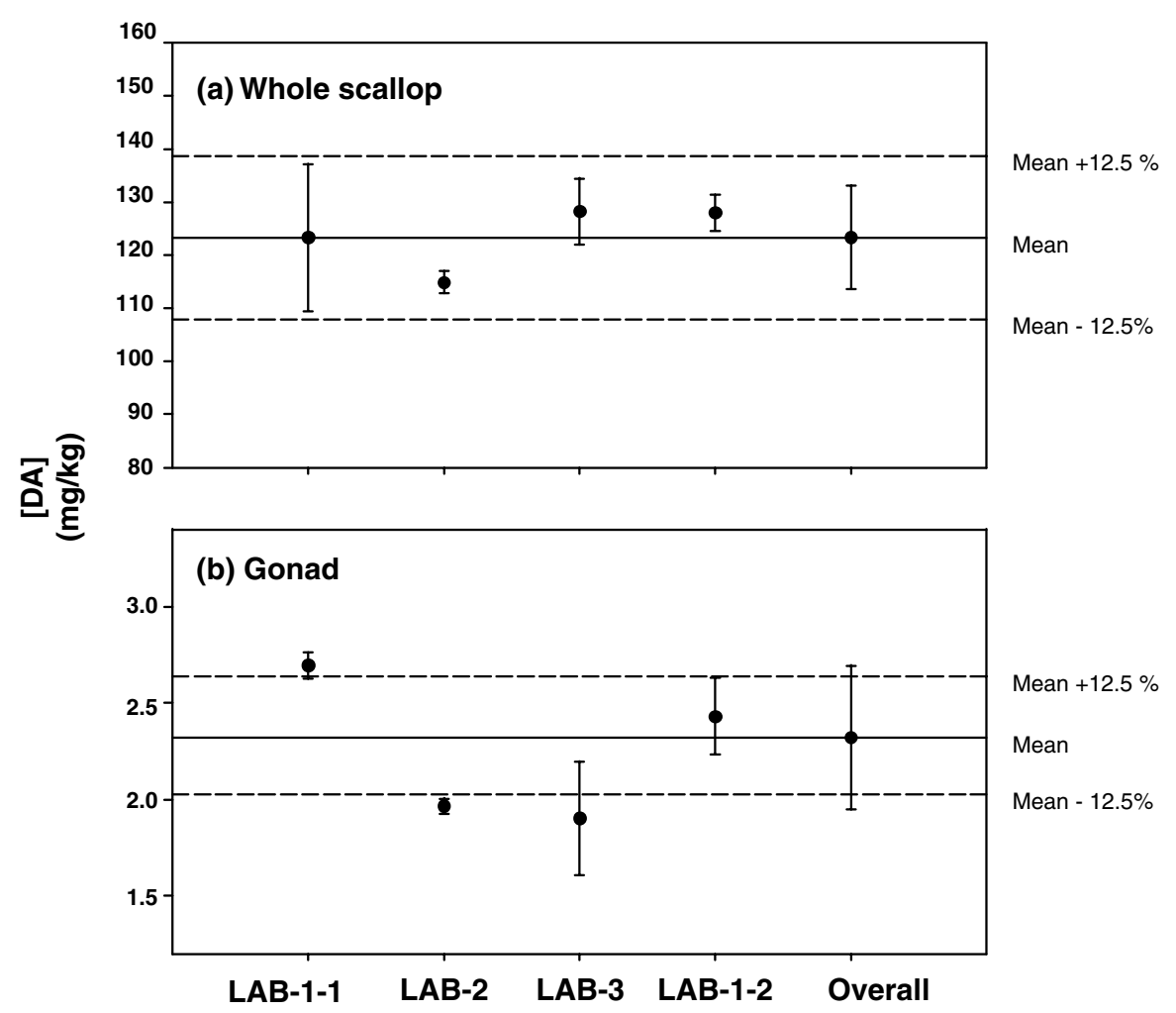

Figure 2. Phase I of study: analysis of (a) whole-scallop-tissue homogenate, and (b) scallop-gonad-tissue homogenate. Error bars represent the standard deviation of the mean.

Table 4. Concentrations of DA and percentage coefficients of variation (c.v., in parenthesis) for the standard solutions, and crude and cleaned-up whole-scallop extracts $(\mu \mathrm{g} / \mathrm{mL})$, and the whole and gonad homogenate matrices $(\mathrm{mg} / \mathrm{kg}$ ) analyzed during phase I of the inter-comparison study using LC-UV detection

\begin{tabular}{|llllll|}
\hline Laboratory & \multicolumn{3}{c|}{ DA concentration and (\% c.v.) } \\
\cline { 2 - 6 } & Standard solution & Crude extract & Cleaned-up extract & Whole-scallop extract & Scallop gonad \\
\hline LAB-1-1 & $2.1(2.0)$ & $20.4(0.52)$ & $15.7(8.9)$ & $123(11.2)$ & $115(1.8)$ \\
LAB-2 & $1.9(3.1)$ & $20.7(1.93)$ & $17.5(11.1)$ & $128(4.9)$ & $1.72(2.6)$ \\
LAB-3 & $1.98(0.3)$ & $20.1(0.89)$ & $16.8(0.6)$ & $128(2.6)$ & $1.99(2.0)$ \\
LAB-1-2 & $1.96(4.8)$ & $20.6(0.51)$ & $16.6(3.5)$ & $(5.0)$ & $2.46(8.1)$ \\
Mean conc. & 1.99 & 20.5 & 16.6 & $(4.4)$ & \\
Mean c.v. $(\%)$ & $(4.2)$ & $(1.3)$ & & & $(16.7)$ \\
\hline \hline
\end{tabular}

homogenate (Fig. 2(a)). Excluding the DA-concentration data derived from the analysis of the scallop gonad sample, over $80 \%$ of the intra- and inter-laboratory c.v.s were $<6 \%$ (Table 4). For the determination of DA in these particular matrices, the performance of the laboratories was deemed satisfactory.

For the analysis of the gonad homogenate, an overall mean c.v. of $16.7 \%$ for the inter-laboratory study was found (Table 4). The mean concentration data from each participant were in the range
1.93-2.72 mg/kg. However, with the exception of concentration data acquired at the conclusion of phase I, all of the c.v. data fell outside the MAE value of $12.5 \%$ (Fig. 2(b)), and the performance of the participants in analyzing this sample was deemed unsatisfactory.

A possible explanation of these results is that the participants were attempting to quantify DA at concentrations close to their lower limits of determination. Also, at the lower concentration, adverse effects of using 


\begin{tabular}{|c|c|c|c|c|c|c|c|c|c|c|}
\hline \multirow[t]{4}{*}{ Laboratory } & \multicolumn{6}{|c|}{ Nominal DA concentration $(\mu \mathrm{g} / \mathrm{mL})$} & & & & \\
\hline & \multicolumn{2}{|c|}{5} & \multicolumn{2}{|c|}{2} & \multicolumn{2}{|c|}{1} & \multirow{2}{*}{\multicolumn{2}{|c|}{$\begin{array}{l}\text { DA + epi-DA in } \\
\text { gonad [mg/kg] }\end{array}$}} & \multirow{2}{*}{\multicolumn{2}{|c|}{$\begin{array}{l}\text { DA + epi-DA in } \\
\text { gonad [mg/kg], } \\
\text { corrected }^{*}\end{array}$}} \\
\hline & \multicolumn{6}{|c|}{ Observed DA concentration $(\mu \mathrm{g} / \mathrm{mL})$} & & & & \\
\hline & Mean & c.v. $(\%)$ & Mean & c.v. $(\%)$ & Mean & c.v. $(\%)$ & Mean & c.v. $(\%)$ & Mean & c.v. $(\%)$ \\
\hline LAB-1-1 & 5.02 & 1.53 & 1.98 & 1.14 & 0.98 & 1.83 & 19.6 & 5.87 & 19.9 & 5.88 \\
\hline LAB-2 & 5.15 & 0.64 & 2.08 & 1.95 & 1.02 & 2.98 & 19.2 & 0.89 & 21.3 & 0.85 \\
\hline LAB-3 & 5.10 & 0.51 & 2.17 & 0.53 & 1.19 & 0.59 & 17.0 & 5.12 & 19.3 & 5.18 \\
\hline LAB-4 (MS) & 4.90 & 0.36 & 1.84 & 0.82 & 0.92 & 0.91 & 18.5 & 7.35 & 23.4 & 7.35 \\
\hline LAB-1 (MS) & 5.03 & 1.72 & 2.01 & 0.92 & 0.98 & 0.96 & 19.4 & 8.30 & 19.7 & 8.17 \\
\hline LAB-1-2 & N/A & N/A & N/A & $\mathrm{N} / \mathrm{A}$ & N/A & $\mathrm{N} / \mathrm{A}$ & 20.7 & 8.84 & 21.0 & 8.86 \\
\hline Overall & 5.04 & 1.9 & 2.01 & 6.0 & 1.02 & 10.0 & 19.1 & 6.5 & 20.8 & 7.2 \\
\hline
\end{tabular}

single-point calibration compared to multi-level calibration curves may have a negative effect on accurate quantification. However, it should be noted where multilevel calibration solutions were used (e.g., by LAB-3), similar concentration data was obtained where a single point curve was applied (e.g., by LAB-2).

These findings suggest that, if DA concentrations of $\sim 2 \mathrm{mg} / \mathrm{kg}$ in gonad samples are to be routinely quantified in an ASP monitoring programme, further research is required to optimize the methodology, unless a higher MAE value (i.e. $>25 \%$ ) is agreed upon. The recent introduction of a trigger level of $4.6 \mathrm{mg} / \mathrm{kg}$ in the monitoring of ASP in scallops [9] also highlights the need for proficiency testing at an international level to demonstrate the performance of official testing laboratories.

\subsection{Assessment of the effect of elution volume on $D A$ recovery using DA CRM}

In phase I, a 19\% reduction in the overall mean DA concentration obtained from all participants was observed after SAX clean-up of the whole-scallop crude extract (Table 4), and this led to an investigation of the recovery efficiency of the clean-up stage. The elution profile for the recovery of DA in an extract of the MUS-1B CRM and using a SAX-cartridge is shown in Fig. 5. Although a large percentage of DA was eluted within the first $2 \mathrm{~mL}$ of elution, a significant percentage remained as a tailing peak. Analysis performed with laboratories using 2- or 3-mL elution volumes resulted in DA recovery from the CRM of $79.0-90.4 \%$, whereas the use of $5 \mathrm{~mL}$ resulted in $98.4 \%$ recovery (Table 6). The participants also used different brands of SAX cartridges, as detailed in Table 2. It is possible that the cartridge sorbent could be a cause of the loss of DA during the extract cleanup stage. This loss can be addressed by decreasing the crude extract loading volume, and by increasing the elution volume. A previous report [4] demonstrates a 93\% recovery using a 2-mL eluent volume compared to the $79.0-90.4 \%$ found with the same volume in the study reported here. Differences in recovery efficiencies using SAX sorbents can occur, and it is recommended that batch-to-batch differences of the same brand should be checked routinely to ensure continuous quality of analysis.

\subsection{Phase II - participant performances}

The quantitation of DA in the standard solutions demonstrated that the overall mean concentrations for the three DA standard solutions were $<2 \%$ different from the respective nominal values (Table 5). The c.v.s for within-laboratory analysis were less than $3 \%$ in all cases, although the inter-laboratory variations were in the range $1.9-10 \%$, and the percentage c.v. values increased as the DA-standard concentration decreased (Table 5).

For the $5-\mu \mathrm{g} / \mathrm{mL}$ DA standard solution, all of the participant results fell within the $12.5 \%$ MAE value (Fig. 3) and were deemed satisfactory.

However, differences were apparent between concentration data generated for the lower standards ( 2 and $1 \mu \mathrm{g} / \mathrm{mL}$ ), and by the participants using UV alone or both UV and MS detectors. Where these data fell outside the MAE value, this suggests problems existed with the preparation of the DA calibration standards at the time of the study.

All regulatory laboratories failed to meet the 25\% MAE value for the detection of DA in the gonad homogenate containing a mean value of $2.28 \mathrm{mg} / \mathrm{kg}$ of DA.

However, all participants achieved satisfactory results in phase II on analyzing a gonad homogenate contain- 

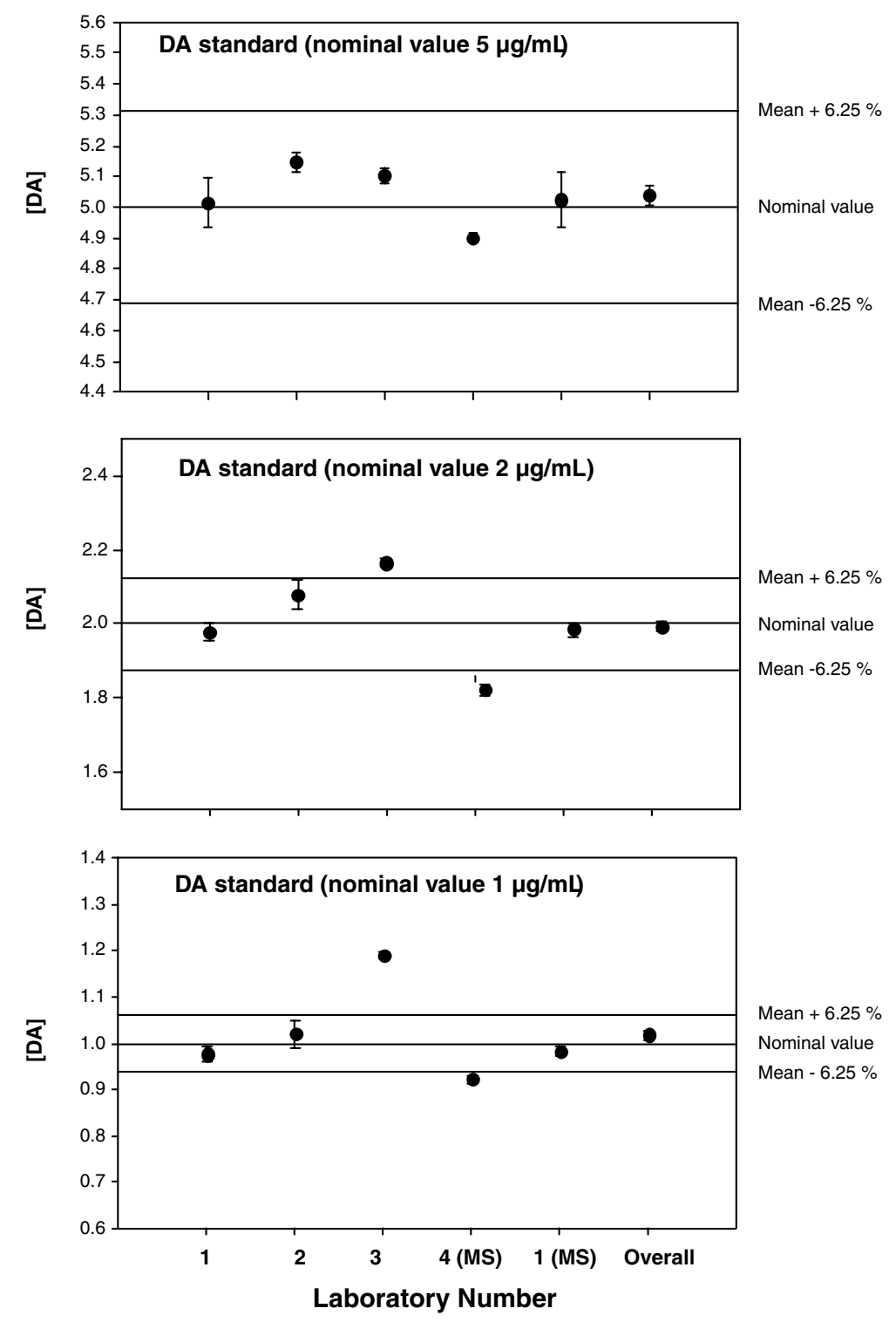

Figure 3. Phase II of study: analysis of three DA standard solutions containing concentrations of 5,2 and $1 \mu \mathrm{g}(\mathrm{DA}) / \mathrm{mL}$, respectively.

ing a level of DA one order of magnitude higher in concentration.

Due to the slightly low DA recoveries obtained from the CRM (Table 6), the results for the gonad homogenate analyzed in phase II were plotted both as the sum of DA and its epimer, epi-DA, concentration before and after recovery correction was applied (Figs. 4(a) and (b), respectively). The average concentration of the gonad homogenate raw data from all participants was $19.1 \mathrm{mg} / \mathrm{kg}$, while the average result for the recoverycorrected data was $20.8 \mathrm{mg} / \mathrm{kg}$. This represents $\sim 8 \%$ negative bias for the uncorrected average between laboratories.

The c.v.s for between laboratories were $6.5 \%$ for data uncorrected for recovery and $7.2 \%$ for recovery- corrected data (Table 5), which were considerably lower than that obtained between participants for gonad homogenate in phase I (16.7\%; Table 4).

A comparison between LC-UV and LC-MS concentration data of the scallop gonad showed no significant difference (Fig. 4).

The study also compared data generated by two LC-MS techniques [LAB-1(MS) and LAB-4] with concentrations obtained by LC-UV detection. The LC-MS method used by LAB-1, which is an adaptation of the method described by Hess et al. [10], gave similar results to the HPLC-UV data for DA standards and gonad homogenate, reinforcing the finding by Hess et al. [10] that the techniques can be equivalent. The fact that the results obtained by two different LC-MS methods were 


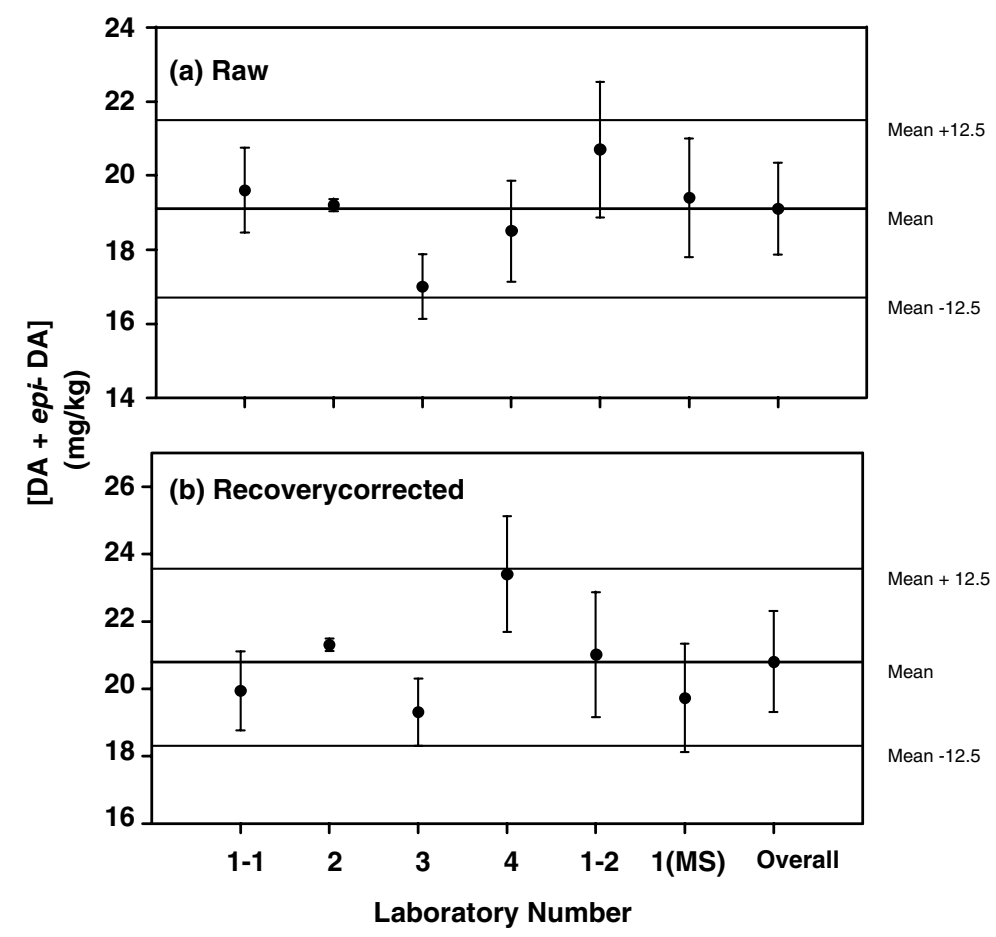

Figure 4. Phase II of study: analysis of a scallop-gonad homogenate showing (a) raw data, and (b) recovery corrected data.

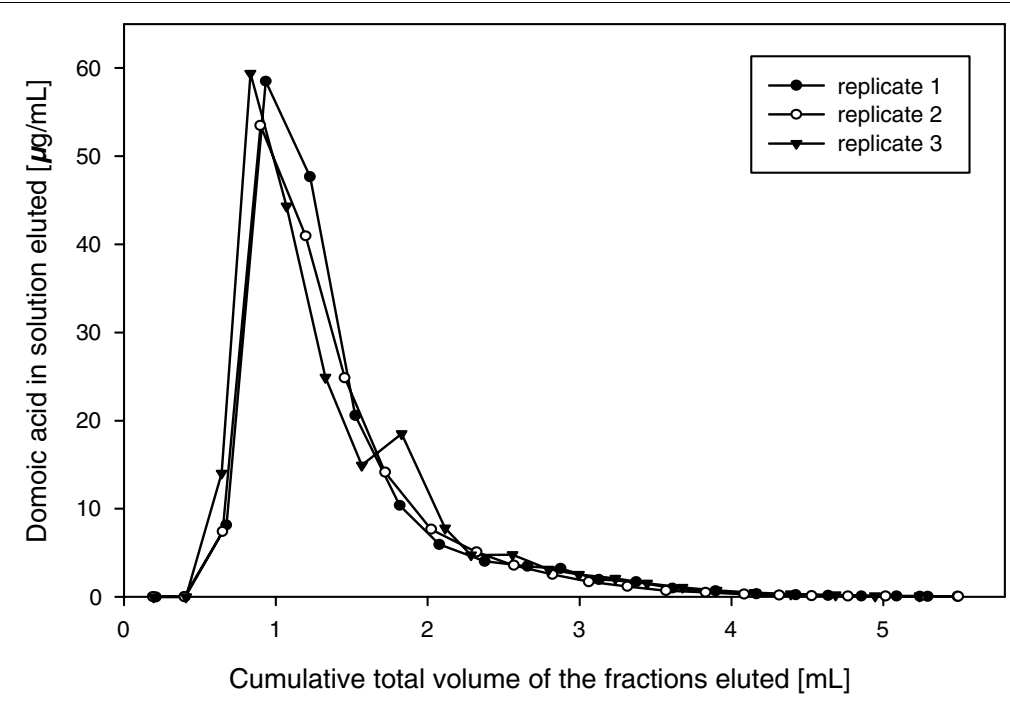

Figure 5. Elution profile of a DA standard solution using a Waters QMA SAX cartridge.

Table 6. The percentage recovery of DA from the CRM (MUS1-B) in relation to the elution volumes applied during clean up

\begin{tabular}{|lll|}
\hline Laboratory & DA recovered (\%) & Elution volume (mL) \\
\hline LAB-1 (phase I) & 89.4 & 2 \\
LAB-1 (phase II) & 98.4 & 5 \\
LAB-2 & 90.4 & 2 \\
LAB-3 & 87.8 & 2 \\
LAB-4 & 79.0 & 3 \\
\hline
\end{tabular}


similar for the gonad homogenate but differed for the DA standards again points towards possible inaccuracies in the preparation of calibration standards.

\section{Summary}

It was apparent that there was a need for laboratories involved with the regulatory monitoring of DA in shellfish to demonstrate comparability of concentration data, since each laboratory had adopted a single published method although there were variations in its deployment. A critical review of the evidence emerging from the inter-comparisons enabled suggestions to be made to improve the confidence of the data. This study was limited to four laboratories, although there was an obvious requirement to extend such exercises to other service laboratories responsible for monitoring ASP toxins in commercial shellfish.

Since this study, inter-comparisons have been initiated under the auspices of the QUASIMEME programme, and have embraced a larger participant base. It is expected that this programme will continue to evolve, providing laboratories with opportunities to assess and refine their methodologies in a manner similar to that reported here. Simultaneously and on a wider scale, it is expected that a trend of improved analytical data will become evident amongst monitoring laboratories responsible for safe- guarding the health of shellfish consumer from toxins, such as domoic acid.

\section{Acknowledgements}

This project was funded by the UK Food Standards Agency, and the Scottish Environment Executive Rural Affairs Department. The authors thank Drs. Ian Davies, Colin Moffat and David Wells of FRS for their comments.

\section{References}

[1] E.C.D. Todd, J. Food Protect. 56 (1993) 69.

[2] European Commission, Council Directive 91/492/EEC of 15 July, Off. J. L 268 (1991) 1.

[3] European Commission, Council Directive 97/61/EC of 20 October, Off. J. L 295 (1997) 35.

[4] M.A. Quilliam, M. Xie, W.R. Hardstaff, J. AOAC Int. 78 (1995) 543.

[5] AOAC International, AOAC Official Method 991.26, in: P. Cunniff (Editor), Official Methods of Analysis of AOAC International, 16th Edition, 3rd Revision, AOAC International, Gaithersburg, MA, USA, 1997, Chapter 35: 9, p. 2.

[6] W.P. Cofino, D.E. Wells, Mar. Pollut. Bull. 29 (1994) 149.

[7] D.E. Wells, W.P. Cofino, Mar. Pollut. Bull. 35 (1997) 18.

[8] ISO/IEC, Guide 43: Proficiency testing by inter-laboratory comparisons, International Standardization Organization, Geneva, Switzerland, 1997.

[9] European Commission, Commission Decision 2002/226/EC, European Commission, Brussels, Belgium, 2002.

[10] P. Hess, S. Gallacher, L.A. Bates, N. Brown, M.A. Quilliam, J. AOAC Int. 84 (2001) 1655. 